\title{
Non-linear effects of pore pressure increase on seismic event generation in a multi-degree-of-freedom rate-and-state model of tectonic fault sliding
}

\author{
Sergey B. Turuntaev ${ }^{1,2,3}$ and Vasily Y. Riga ${ }^{2}$ \\ ${ }^{1}$ Institute of Geosphere Dynamics, Russian Academy of Sciences, Moscow, 119334, Russian Federation \\ ${ }^{2}$ Moscow Institute of Physics and Technology, Moscow, 141701, Russian Federation \\ ${ }^{3}$ All-Russian Research Institute of Automatics, Moscow, 127055, Russian Federation
}

Correspondence to: Sergey B. Turuntaev (s.turuntaev@gmail.com)

Received: 1 November 2016 - Discussion started: 12 December 2016

Revised: 28 March 2017 - Accepted: 29 March 2017 - Published: 22 May 2017

\begin{abstract}
The influence of fluid injection on tectonic fault sliding and seismic event generations was studied by a multidegree-of-freedom rate-and-state friction model with a twoparametric friction law. A system of blocks (up to 25 blocks) elastically connected to each other and connected by elastic springs to a constant-velocity moving driver was considered. Variation of the pore pressure due to fluid injection led to variation of effective stress between the first block and the substrate. Initially the block system was in a steady-sliding state; then, its state was changed by the pore pressure increase. The influence of the model parameters (number of blocks, spring stiffness, velocity weakening parameter) on the seismicity variations was considered. Various slip patterns were obtained and analysed.
\end{abstract}

\section{Introduction}

Despite the fact that the rate-and-state model of friction was proposed in the second half of the previous century, the interest in it has increased in recent years. The rate-and-state model (Gu et al., 1984; Dieterich, 1992; Abe and Kato, 2013) was adopted as a quite appropriate basis for describing seismic processes in the Earth's crust and for modelling relevant geophysical systems. Currently, it is believed that this model describes the seismic process most adequately.

Brace and Byerlee (1966) proposed considering unstable frictional sliding along tectonic faults as a model of earthquakes. The model included a suggestion that a cohesion ex- isting in some parts of tectonic fault prevents free slipping along it and leads to an accumulation of a shear stress to a critical level, after which the slip and the earthquake occur.

Peculiarities of the friction force dependence on the duration of the stationary state of the contact and on the velocity of the motion along the fault were examined by Dieterich (Dieterich, 1992). Gu et al. (1984) experimentally investigated various modes of the frictional movements and determined empirical constants whose values are used in many modern variants of the rate-and-state equation.

The rate-and-state equation was considered by Hobbs (Hobbs, 1990) by means of non-linear dynamics methods. Change in the friction was studied as a function of displacement and velocity at a variation of the stiffness coefficient in the rate-and-state equation. A similar approach was implemented by Erickson et al. (2008); they examined an appearance of chaotic solutions in the one-parameter velocitydependent friction equation.

Abe and Kato (Abe and Kato, 2013, 2014) examined two- and three-degree-of-freedom spring-block models with a one-parameter rate-and-state friction law and obtained different slip patterns for such system. By varying stiffness parameters, they obtain periodic recurrence of the seismic and aseismic events and several types of seismicity chaotic behaviour.

Turuntaev et al. (2012) showed using the GrassbergerProcaccia method (Grassberger and Procaccia, 1983) that the man-made impact underground leads to an increase in the "regularity" of the seismic regime. To explain the increase 


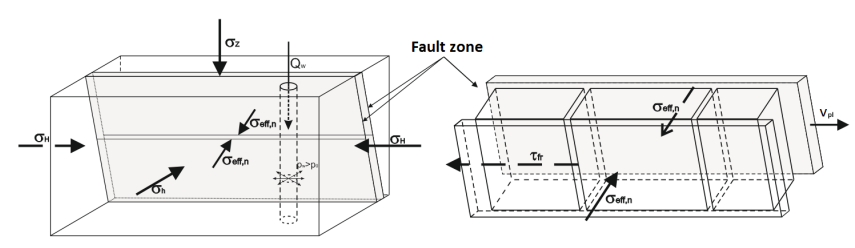

(a)

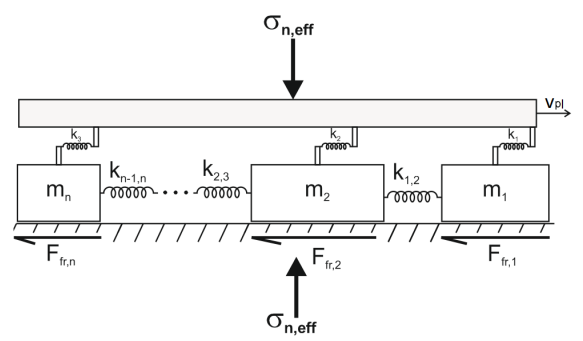

(b)

Figure 1. (a) The block model of an active tectonic fault; (b) schematic diagram of a multi-degree-of-freedom spring-block model.

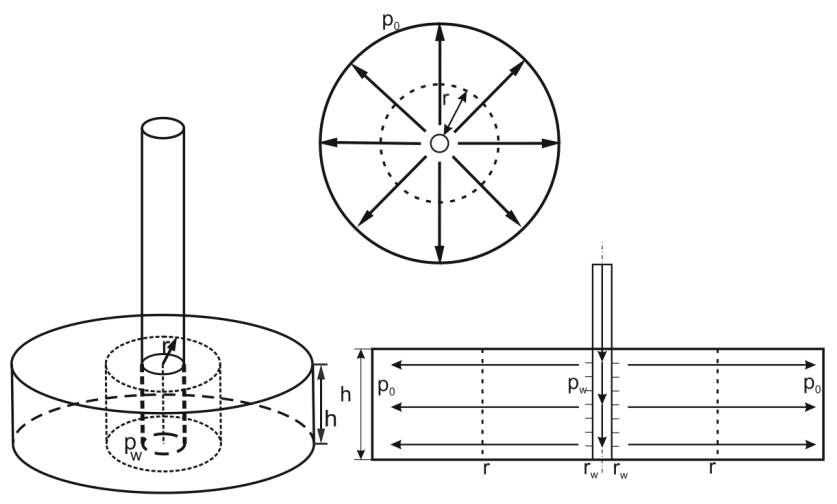

Figure 2. Radial flow in a homogeneous reservoir.

in the seismic regime regularity, a model of the fault motion defined by the two-parameter velocity dependent friction law was considered.

In the presented paper, we consider the two-parameter type of the friction law in a multi-degree-of-freedom spring-block model and change the value of critical shear stress in the rate-and-state equation, suggesting that this is the value varied by human impact (by fluid injection and corresponding pore pressure change). Here we use the classical pore-elastic model of radial filtration of injected fluid to calculate the typical pore pressure change.

\section{The model description}

\subsection{Spring-block model}

The tectonic fault model proposed by Burridge and Knopov (Burridge and Knopov, 1966) looks like a system of blocks elastically connected to each other (Fig. 1a, b). Each block

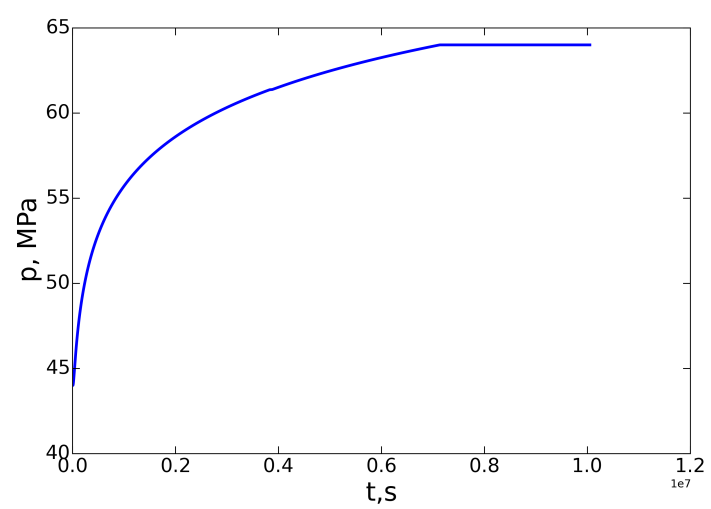

Figure 3. Pore pressure change at the boundary between the first block and substrate.

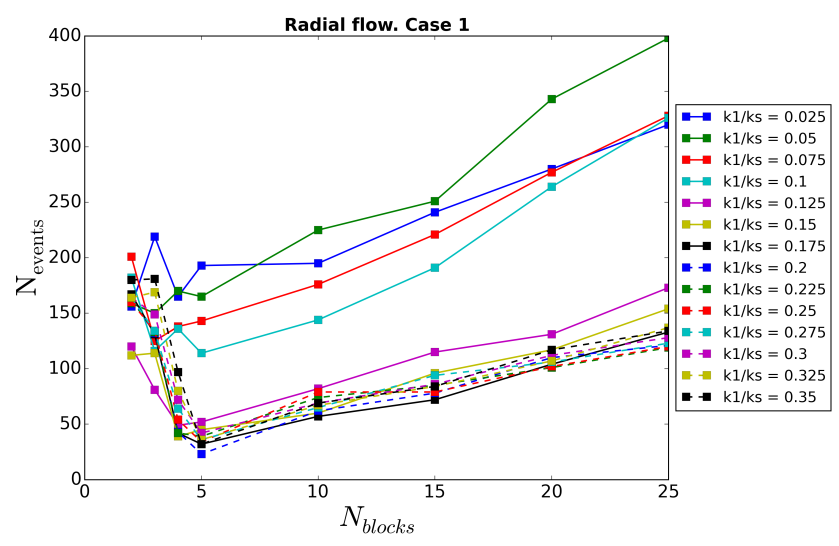

Figure 4. Cumulative number of events vs. number of blocks.

moves under the net action of elastic forces from adjacent blocks and driver and friction force from the stationary substrate. Here, the multi-degree-of-freedom system is investigated. Every block of mass $m_{i}$ is connected by a spring of stiffness $k_{l}$ to the driver moving at a rate $v_{p l}$, and linked with each other by springs of stiffness $k_{n-1, n}$. The motion equation may be written as Eq. (1):

$\left\{\begin{array}{l}m_{1} \ddot{x}_{1}=k_{1}\left(v_{p l} t-x_{1}\right)-k_{12}\left(x_{1}-x_{2}\right)-F_{f r 1}, \\ m_{2} \ddot{x}_{2}=k_{2}\left(v_{p l} t-x_{2}\right)+k_{12}\left(x_{1}-x_{2}\right)-k_{23}\left(x_{2}-x_{3}\right)-F_{f r 2}, \\ \ldots\end{array}\right.$

where $F_{\text {fri }}=S_{i} \tau_{i}$ is the force of friction between the block number $i$ and the substrate, $S_{i}$ is the area of the block surface, $\tau_{i}$ is the shear stress, $t$ is time and $x_{i}$ is the displacements of the blocks relative to the driver.

We assume that the friction shear stress at the block boundary obeys the following two-parameter friction law: 


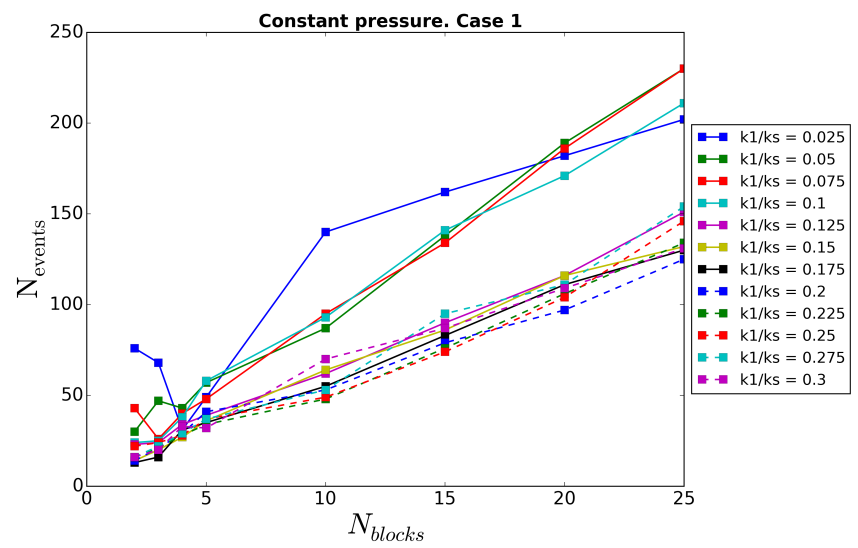

Figure 5. Cumulative number of events vs. number of blocks.

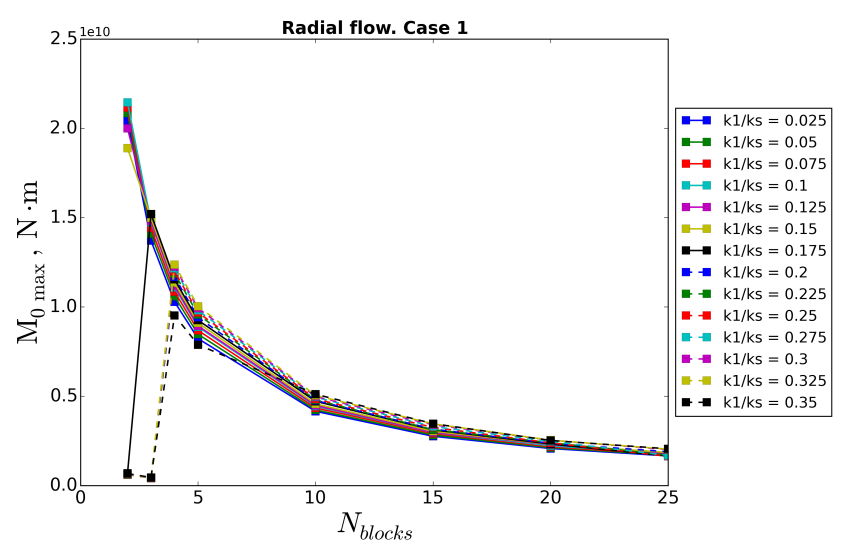

Figure 8. Maximum seismic moment of event vs. number of blocks.

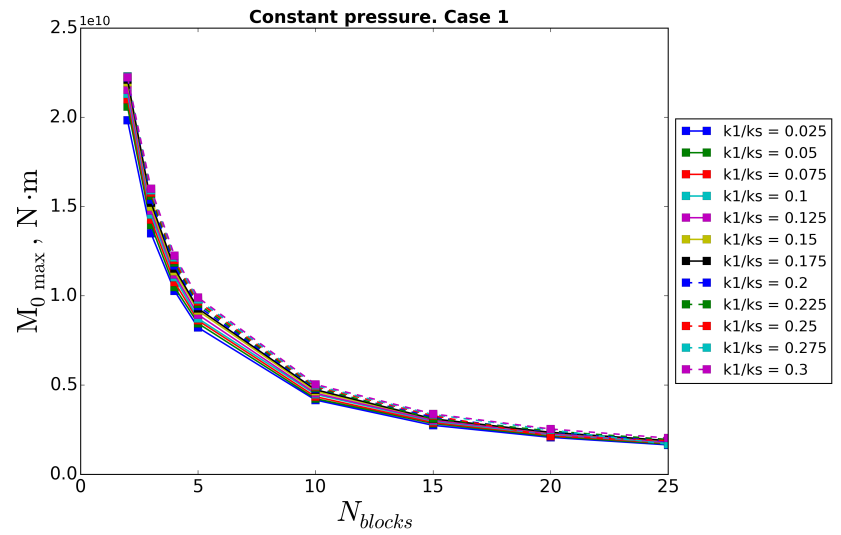

Figure 9. Maximum seismic moment of event vs. number of blocks.

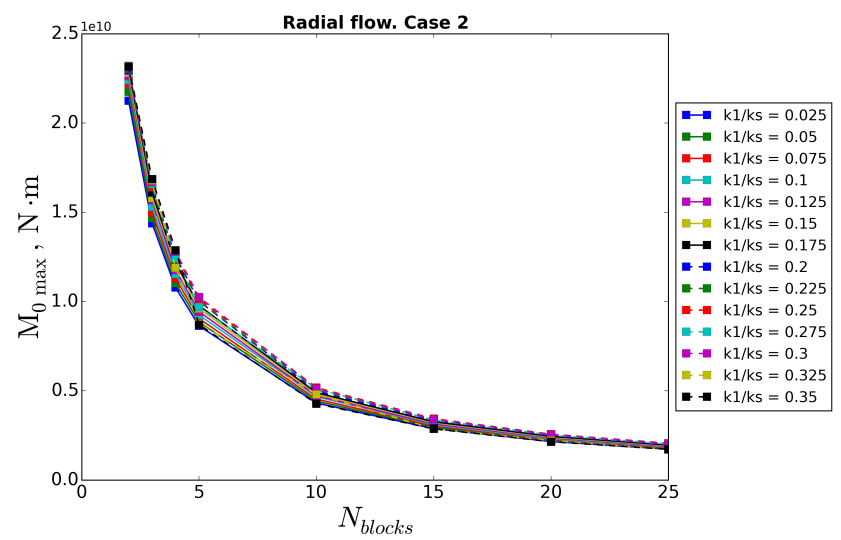

Figure 10. Maximum seismic moment vs. number of blocks.

Figure 7. Cumulative number of events vs. number of blocks. 


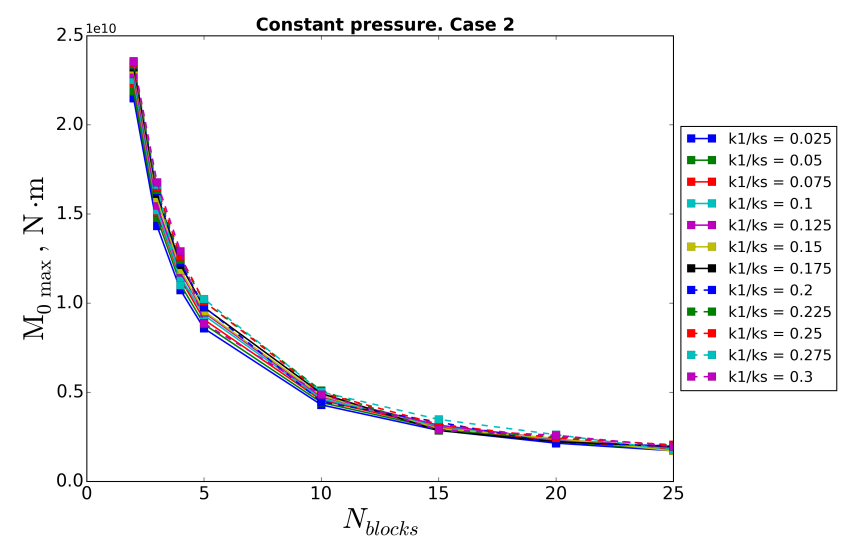

Figure 11. Maximum seismic moment vs. number of blocks.

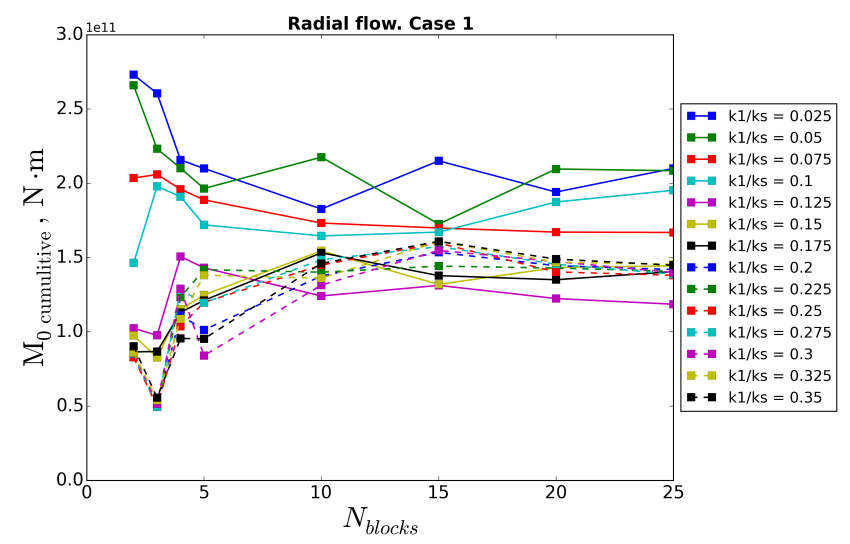

Figure 12. Cumulative seismic moment vs. number of blocks.

$\tau=\tau^{*}+A \ln \left(\frac{v}{v^{*}}\right)+\theta_{1}+\theta_{2}$,

$\tau^{*}=\tau_{0}+\mu\left(\sigma_{\mathrm{n}}-p\right)$,

$\dot{\theta}_{i}=-\frac{v}{L_{i}}\left[\theta_{i}+B_{i} \ln \left(v / v^{*}\right)\right]$,

where $\theta_{1}, \theta_{2}$ are the state parameters, $A$ and $B_{1}, B_{2}$ are constants that represent the rate and the time dependences of the friction, respectively, $L_{1}, L_{2}$ are characteristic slip distances, $v *$ is a reference velocity, $\sigma_{\mathrm{n}}$ is a normal stress, $p$ is fluid pore pressure, $\tau_{0}$ is a cohesion, $\mu$ is the Coulomb friction coefficient, and $\tau *$ is a critical shear stress. Here, the values of constants $A, B_{i}$, and $L_{i}$ were taken from the experiments of Gu et al. (1984).

As was shown by $\mathrm{Gu}$ et al. (1984), if $A-B_{1}-B_{2}<0$, the friction shows velocity weakening, which can lead to stick-slip motion; otherwise, if $A-B_{1}-B_{2} \geq 0$, the friction shows velocity strengthening. For the single-degree-offreedom spring-block model with the spring stiffness $k$, the so-called critical stiffness $k_{\mathrm{cr}}$ (per unit area of block surface) is defined by Eq. (5):

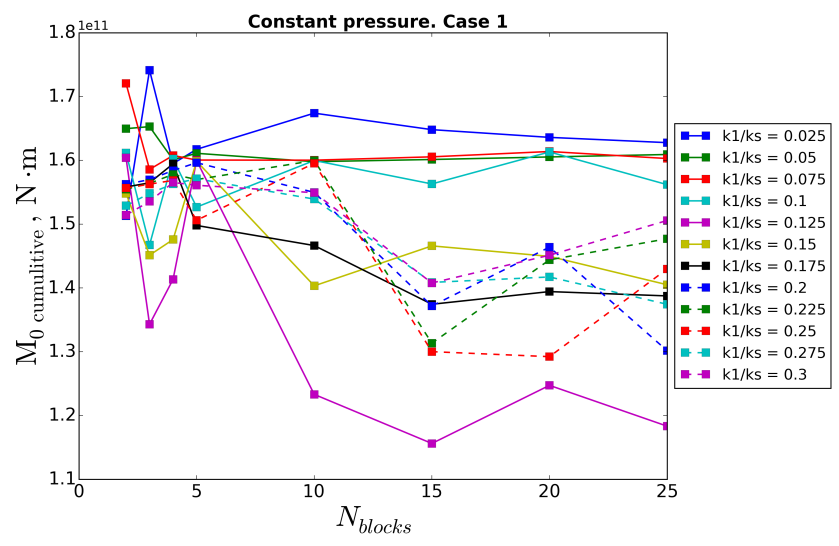

Figure 13. Cumulative seismic moment vs. number of blocks.

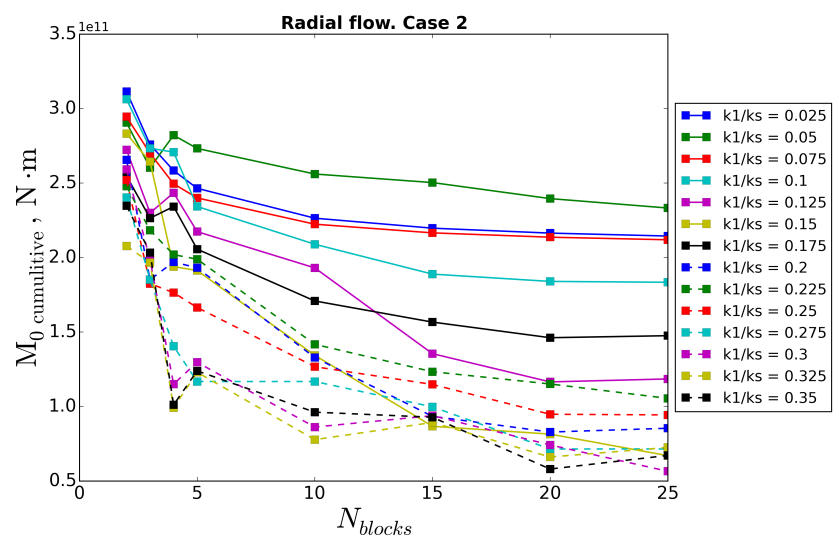

Figure 14. Cumulative seismic moment vs. number of blocks.

$k_{\mathrm{cr}}=\frac{2 A}{L_{1}+L_{2}}\left[\left(\beta_{1}-1\right)+\rho^{2}\left(\beta_{2}-1\right)+2 \rho\left(\beta_{1}+\beta_{1}-1\right)\right.$
$\left.+\sqrt{\left\{\left[\left(\beta_{1}-1\right)+\rho^{2}\left(\beta_{2}-1\right)\right]^{2}+4 \rho^{2}\left(\beta_{1}+\beta_{1}-1\right)\right\}}\right] /(4 \rho)$

where $\beta_{1}=\frac{B_{1}}{A}, \beta_{2}=\frac{B_{2}}{A}$, and $\rho=\frac{L_{1}}{L_{2}}$.

If $k<k_{c r}$ and $A-B_{1}-B_{2}<0$, the stick-slip occurs. Let us suppose that all the blocks have the same friction parameters and stiffness, and that these parameters satisfy the conditions for stick-slip. Initially, all blocks are moving with the velocities equal to the driver velocity. To study the difference between the injection-induced seismicity and the natural seismicity, two sets of numerical calculations were conducted (Set 1 and Set 2). In the first set ("natural" seismicity case), a perturbation in the form of an instant increase in the first block velocity was introduced equal to the velocity of the driver (as considered by Hobs, 1990). In the second set, the pore pressure in the boundary between the first block and the substrate was increased with time in accordance with the pore-elastic equation solution. 


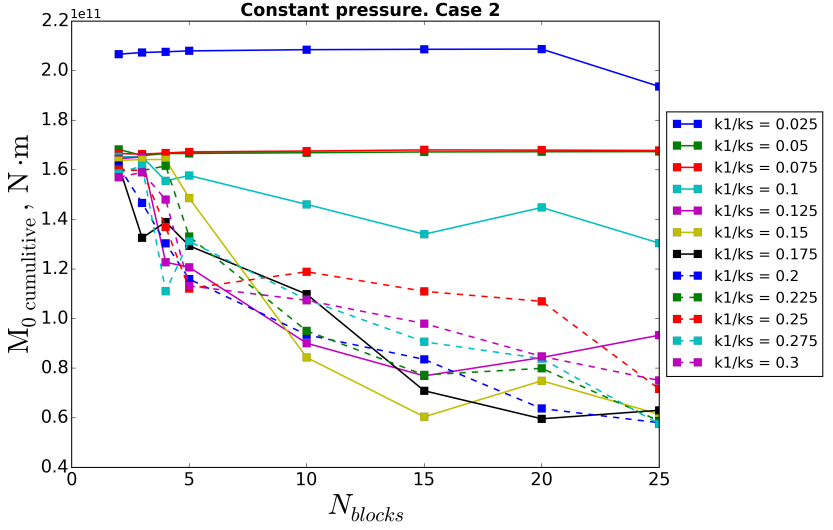

Figure 15. Cumulative seismic moment vs. number of blocks.

Table 1. Values of coefficients $A$ and $k_{\mathrm{cr}}$ used in calculations.

\begin{tabular}{lcc}
\hline Case & $A$ & $k_{\text {cr }}$ \\
\hline 1 & $3.3 \times 10^{4} \mathrm{~Pa}$ & $1.06 \times 10^{10} \mathrm{~Pa} \mathrm{~m}^{-1}$ \\
2 & $3.2 \times 10^{4} \mathrm{~Pa}$ & $1.11 \times 10^{10} \mathrm{~Pa} \mathrm{~m}^{-1}$ \\
\hline
\end{tabular}

The parameters in all the simulations were the following: $B_{1}=3.3 \cdot 10^{4} \mathrm{~Pa}, B_{2}=2.772 \cdot 10^{4} \mathrm{~Pa}, L_{1}=2.5$. $10^{-7} \mathrm{~m}, L_{2}=5.2 \cdot 10^{-6} \mathrm{~m}, v_{p l}=10^{-9} \mathrm{~m} \mathrm{~s}^{-1}\left(3.2 \mathrm{~cm} \mathrm{yr}^{-1}\right)$, $k_{s}=9.04 \cdot 10^{9} \mathrm{~Pa} \mathrm{~m}^{-1}$ (stiffness per unit area of the block), $\tau^{*}=99 \mathrm{MPa}$; the block mass obeyed the condition $\frac{m v_{p l}^{2}}{A S} \ll$ 1; $S$ was the area of the block contact with the substrate. By using such a small mass, we can neglect the inertness of the system and Eq. (5) will be relevant for our system. For both sets of the calculations, two cases were considered, which differed by the values of $A$ and $k_{\mathrm{cr}}$ (Table 1). It was shown (Gu et al., 1984; Hobbs, 1990) that the one-block system will move chaotically in Case 1 and periodically in Case 2.

\subsection{Pore pressure change}

To estimate the pore pressure change, we considered radial flow of fluid in an infinite homogeneous reservoir of constant thickness from the injection well with a negligibly small radius (Fig. 2). The initial reservoir pressure was assumed to be the same everywhere and equal to $p_{0}$. The volumetric flow rate from the well was constant and equal to $Q_{0}$. The assumptions were the following: the permeability was constant (independent of the pressure), and the fluid had small and constant compressibility. To express the condition for constant flow rate $u_{r}$ at the well bore, Darcy's law was used:

$$
\begin{aligned}
u_{r} & =-\frac{k}{\mu} \frac{\mathrm{d} p}{\mathrm{~d} r}, \\
Q_{0} & =2 \pi h r \cdot u_{r}=-\frac{2 \pi h k}{\mu} r \frac{\mathrm{d} p}{\mathrm{~d} r} .
\end{aligned}
$$

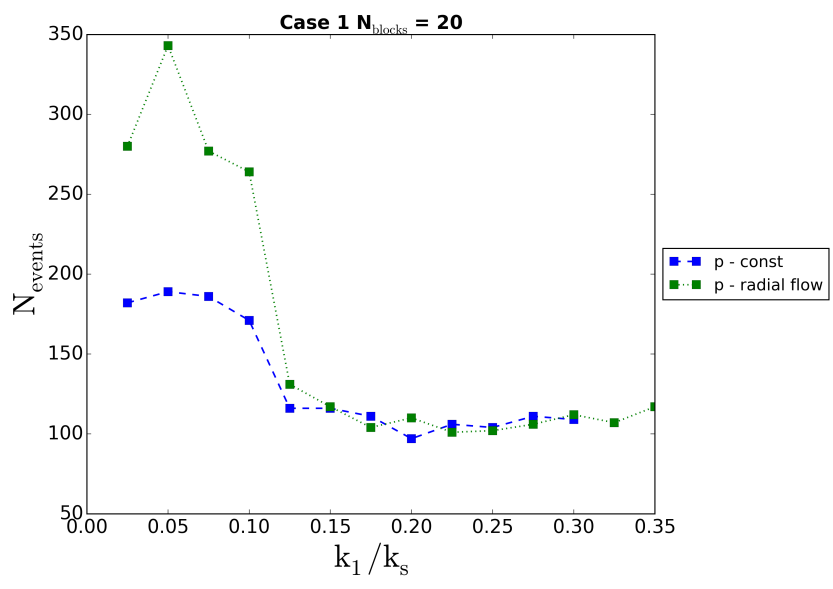

Figure 16. Event cumulative number dependence on the stiffness of the interblock link.

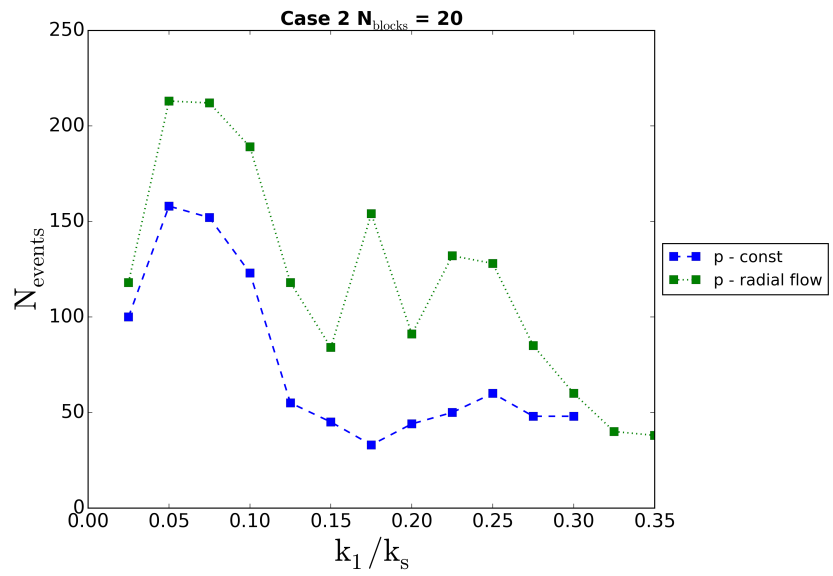

Figure 17. Event cumulative number dependence on the stiffness of the interblock link.

So we got the standard diffusivity equation, where $D=$ $\frac{k}{\varphi \mu c}$ is the hydraulic diffusivity (Matthews and Russel, 1967):

$$
\left\{\begin{array}{l}
\frac{\partial p(r, t)}{\partial t}=D\left(\frac{\partial^{2} p(r, t)}{\partial r^{2}}+\frac{1}{r} \frac{\partial p(r, t)}{\partial r}\right), \\
Q_{0}=-\left.\frac{2 \pi h k}{\mu} r \frac{d p}{d r}\right|_{r=r_{w}},\left(r_{w} \rightarrow 0\right), \\
p(+\infty, t)=p_{0}, \\
p(r, 0)=p_{0} .
\end{array}\right.
$$

The solution of this equation with the above initial and boundary conditions reads as 


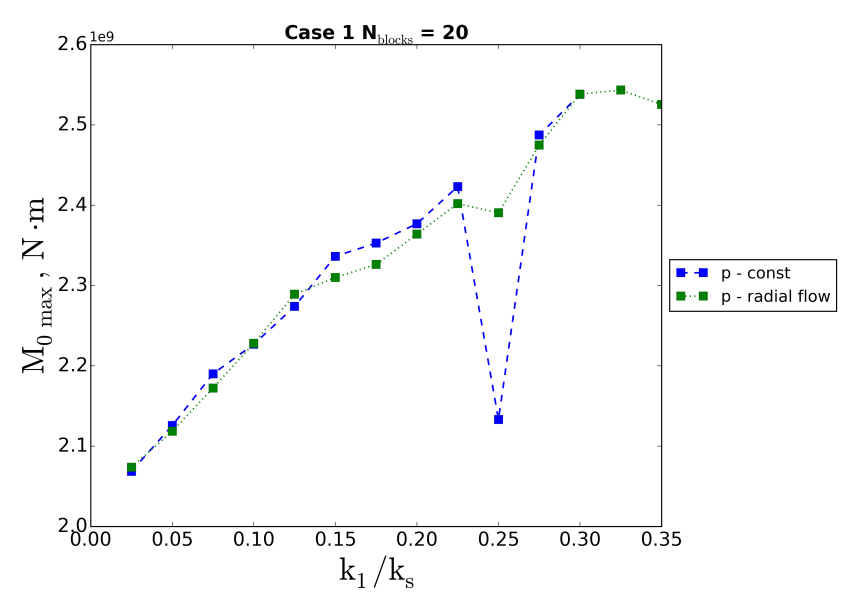

Figure 18. The event maximum seismic moment dependence on the stiffness of the interblock link.

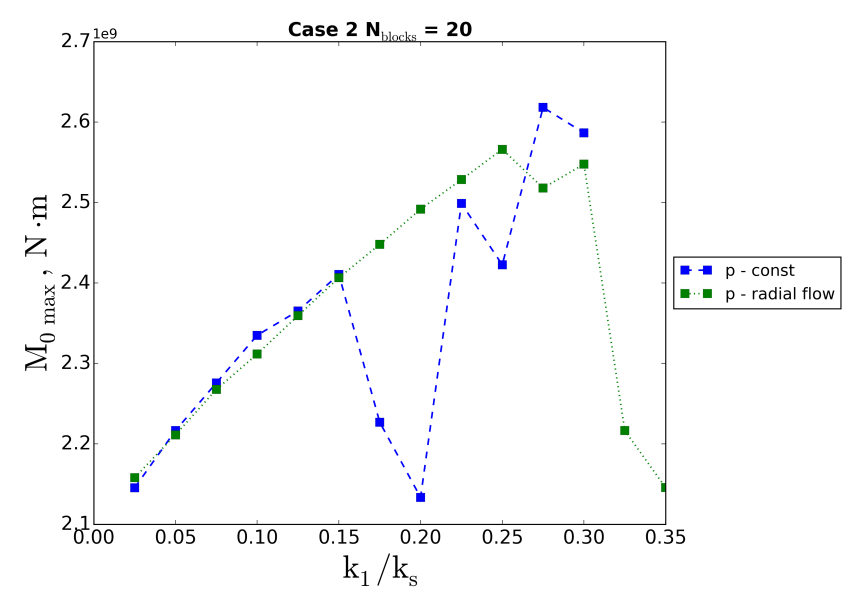

Figure 19. The event maximum seismic moment dependence on the stiffness of the interblock link.

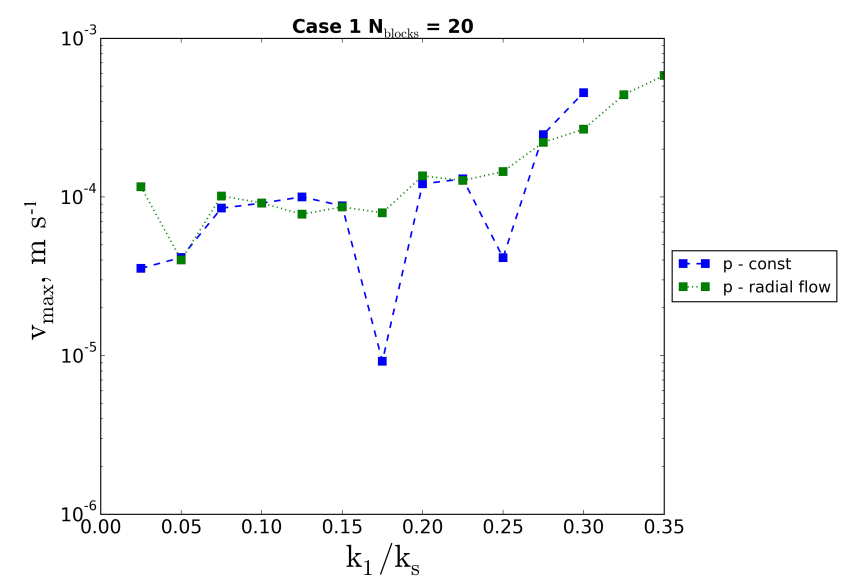

Figure 20. Maximum velocity dependence on the stiffness of the interblock link.

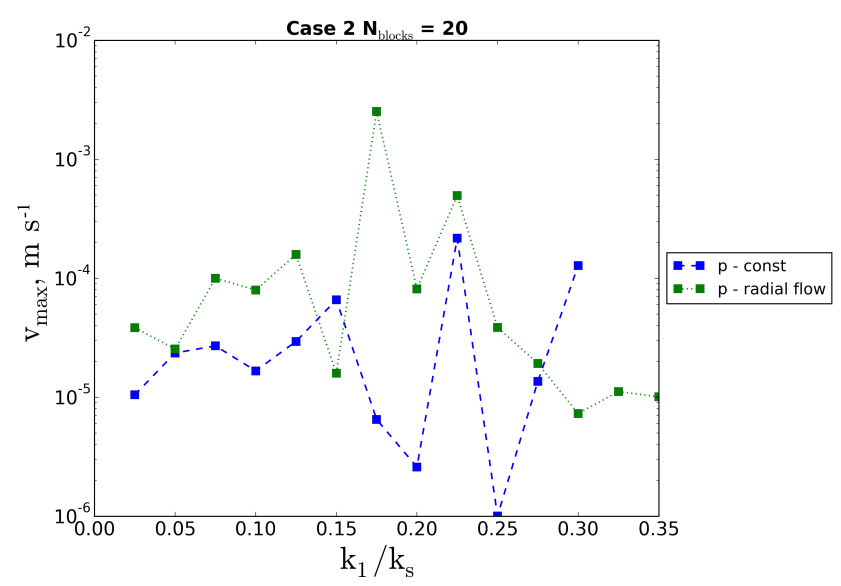

Figure 21. Maximum velocity dependence on the stiffness of the interblock link.

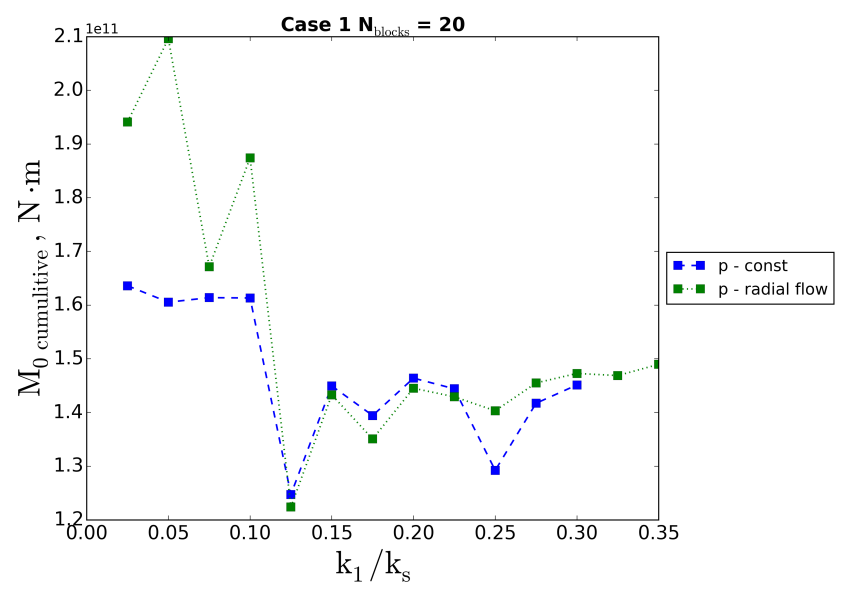

Figure 22. The block system cumulative seismic moment dependence on the stiffness of the interblock link.

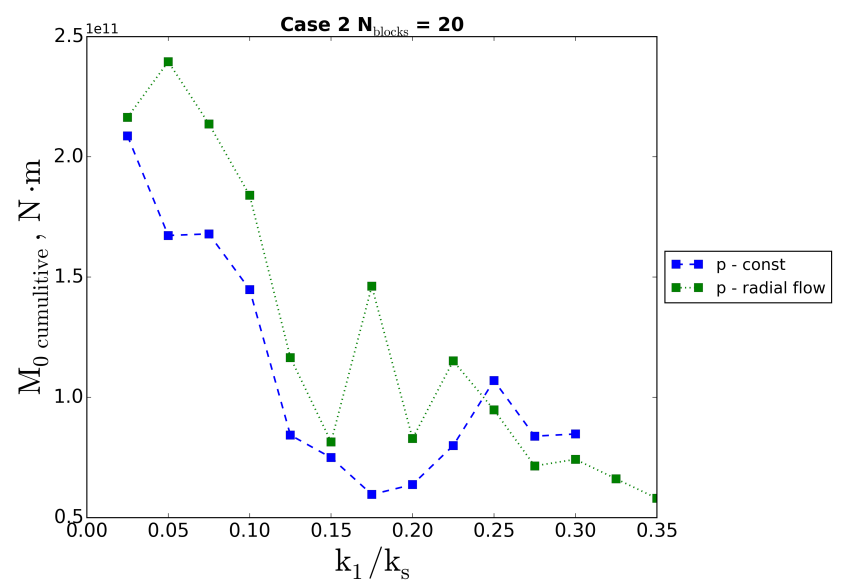

Figure 23. The block system cumulative seismic moment dependence on the stiffness of the interblock link. 


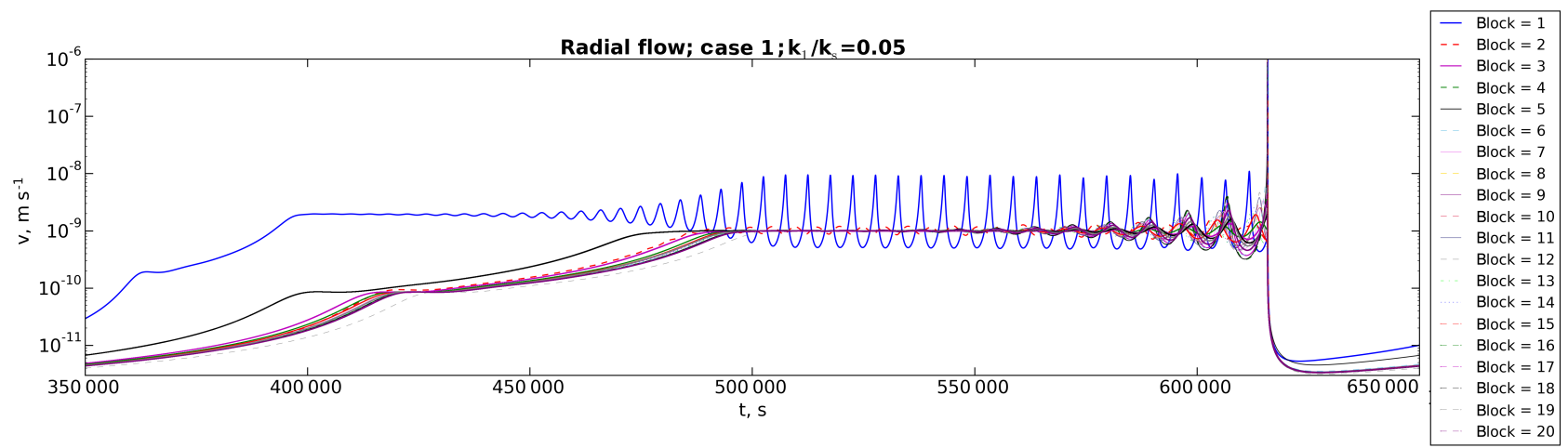

Figure 24. Block velocity variations in time for the system consisting of 20 blocks in Case 1 .

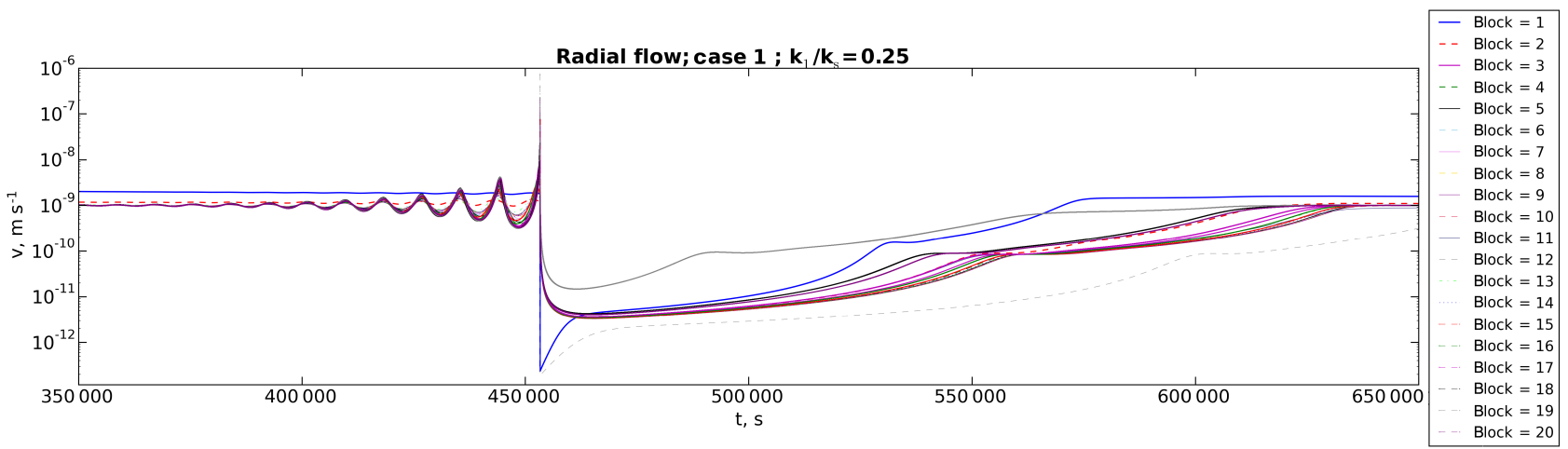

Figure 25. Block velocity variations in time for the system consisting of 20 blocks in Case 1 .

$p=\frac{Q_{0} \mu}{4 \pi k h} E i\left(\frac{r^{2}}{4 D t}\right)+p_{0}$,

$E i(t)=\int_{x}^{\infty} \frac{e^{-t}}{t} \mathrm{~d} t$.

The values of parameters used in the calculations were close to the parameters of the Basel project (Häring et al., 2008): $r=100 \mathrm{~m}, Q_{0}=1.5 \mathrm{~m}^{3} \mathrm{~min}^{-1}, \quad p_{0}=44 \mathrm{MPa}$, $\mu=0.284 \mathrm{~Pa} \mathrm{~s}, \quad h=46 \mathrm{~m}, \quad k=4 \mathrm{mD}, \quad D=0.065 \mathrm{~m}^{2} \mathrm{~s}^{-1}$ (Dinske, 2010). We stopped the pressure growth at the first block boundary when it exceeded the value $64 \mathrm{MPa}$ (the corresponding time is approximately $7.13 \cdot 10^{6} \mathrm{~s}$, Fig. 3). Instead of exponential integral $E i(9)$ we used its approximation (Abramovitz and Stigan, 1979):

$E i(x)=\left\{\begin{array}{l}-\ln \left(\gamma_{1} x\right) \quad 0<x \leq 0,01 \\ -\ln \left(\gamma_{1} x\right)+a_{1} x+a_{2} x^{2}+a_{3} x^{3}+a_{4} x^{4}+a_{5} x^{5}, \\ 0,01<x \leq 1 \\ \left(\frac{x^{2}+b_{1} x+b_{2}}{x^{2}+c_{1} x+c_{2}}\right) \frac{e^{-x}}{x} \quad 1<x<+\infty\end{array}\right.$

where $a_{1}=0.99999193 ; \quad a_{2}=-0.24991055 ; \quad a_{3}=$ $0.05519968 ; a_{4}=-0.00976004 ; a_{5}=0.00107857 ; b_{1}=$
$2.334733 ; b_{2}=0.250621 ; c_{1}=3.330657 ; c_{2}=1.681534 ;$ $\gamma_{1}=1.7810$.

\section{Results}

To study the influence of the number of blocks in the multidegree-of-freedom spring-block system on characteristics of the simulated seismicity (the total number of events, the maximum and cumulative seismic moments) for the "natural" and "induced" cases, the calculations for 2, 3, 4, 5, 10, 15, 20 and 25 blocks were made for the same motion durations - 1 million seconds. The time restriction was related to the computational complexity of 25 block simulations. During this time, the pressure changed significantly in Set 2 (near $11 \mathrm{MPa}$ ). The number of events, the maximum seismic moment of one event and the cumulative seismic moment of all events and all blocks are shown in Figs. 4-15. The calculations were made for different ratios of stiffness of the springs between the blocks $k_{1}$ to stiffness of links between the driver and the blocks $k_{s}$.

It can be seen that if the pore pressure did not change (Set 1, Figs. 5, 7, 9, and 11), the number of events grows almost linearly with the increase in the number of blocks for all values of stiffness of springs between the blocks in both cases 


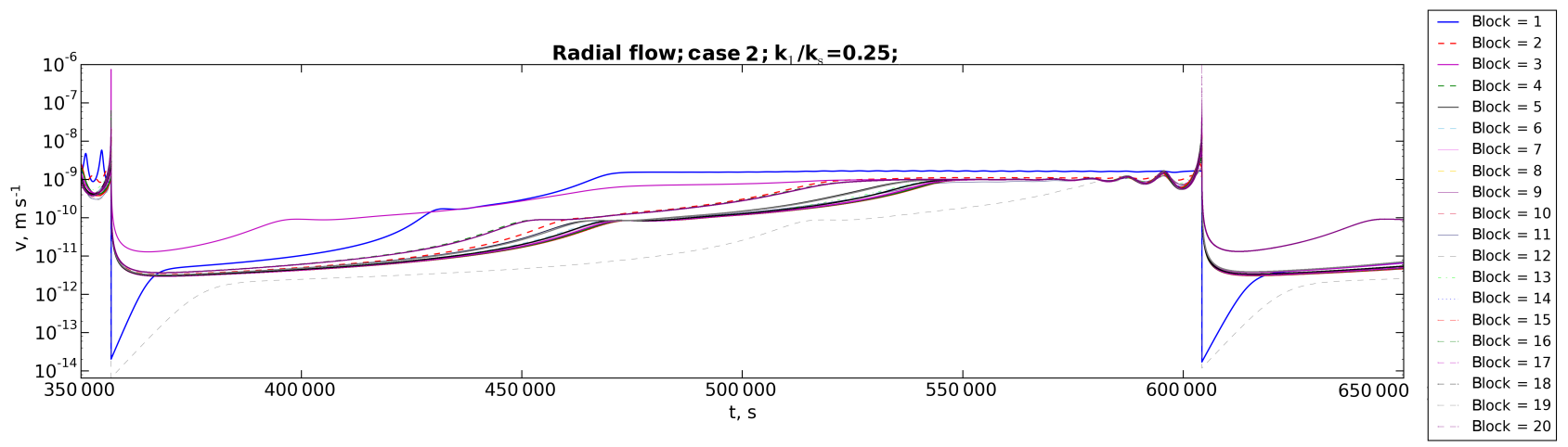

Figure 26. Block velocity variations in time for the system consisting of 20 blocks in Case 2 .

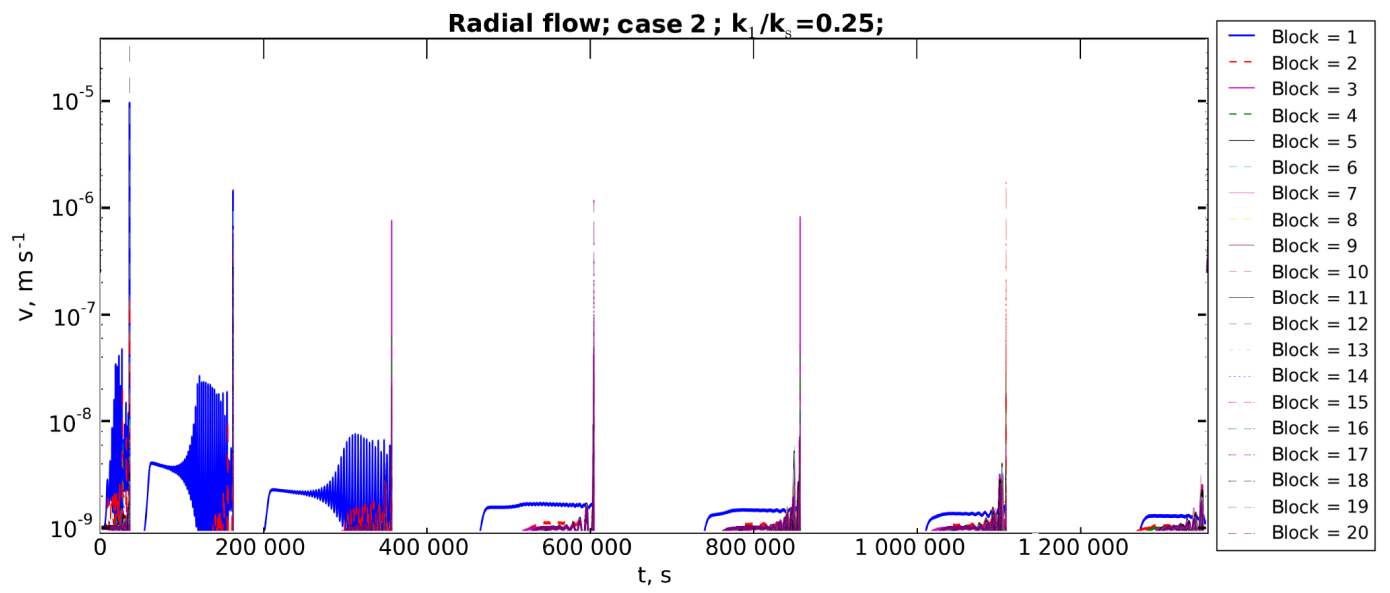

Figure 27. Seismic event occurrences in time for the system consisting of 20 blocks in Case 2.

(1 and 2); the maximum seismic moment of the events decreases with the increase in the number of blocks.

However, for small values of $k_{1}$ (equal to $0.025 k_{s}$ ) the total seismic moment does not depend on the number of blocks for both cases. In Case 1 and $k_{1}>0.1 k_{s}$ for $N_{\text {blocks }} \leq 10$ the cumulative seismic moment slightly decreases; for $N_{\text {blocks }}>10$ it almost does not change. In Case 2 the cumulative seismic moment decreases with an increase in the number of blocks. For Set 2, when the pore pressure increases (Figs. 4, 6, 8, and 10), the dependence is more complicated: in Case 1 for $k_{1} \leq 0.1 k_{s}$ the number of events also grows linearly with the increase in the number of blocks, but for $0.1 k_{s}<k_{1} \leq 0.35 k_{s}$ the number of events decreases with the increase in the number of blocks up to 5, and only then does it start to increase linearly. The maximum seismic moment decreases in both cases; the deviation of one point in Set 2 ("induced" seismicity simulation) from the main trend is caused by insufficient calculating time. The total seismic moment almost does not change in Case 1, and gradually decreases in Case 2.

Now, let us consider the change in the behaviour of the system consisting of 20 blocks with the change in the stiffness of the link between the blocks $k_{1}$. In Case 1 (both sets, Figs. 16,
18, and 20), the total number of events initially decreases with the increase in $k_{1}$ and then stabilizes at a value around 100 , while the maximum seismic moment and the maximum block velocity increase almost monotonically. These results can be explained by the following. Case 1 corresponds to the chaotic behaviour of the one-block system; the characteristic feature of that behaviour is the quick changes in the block velocity. If there are many blocks, the interaction of one block with its neighbours prevents a significant increase in the block velocity. At low values of $k_{1}$ every neighbouring block reacts with time lag to movement of the first block, and all blocks move asynchronously and disturb each other. The same effect causes a large number of events. With an increase in $k_{1}$, the first block perturbation transfers faster to other blocks; the system starts moving more synchronously, which leads to the increase in the block velocities and in the event seismic moments. At the same time, the total number of perturbations experienced by each block decreases, which leads to the decrease in the number of events. All these features are illustrated in Figs. 24 and 25. For convenience, we consider a short period of time and truncate the maximum value of the velocity. 


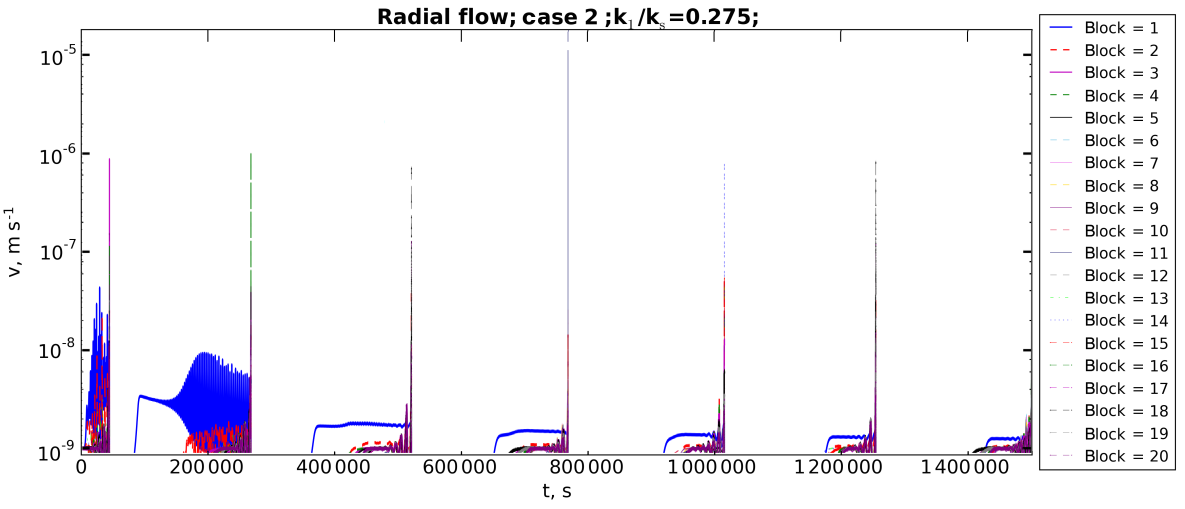

Figure 28. Seismic event occurrences in time for the system consisting of 20 blocks in Case 2.

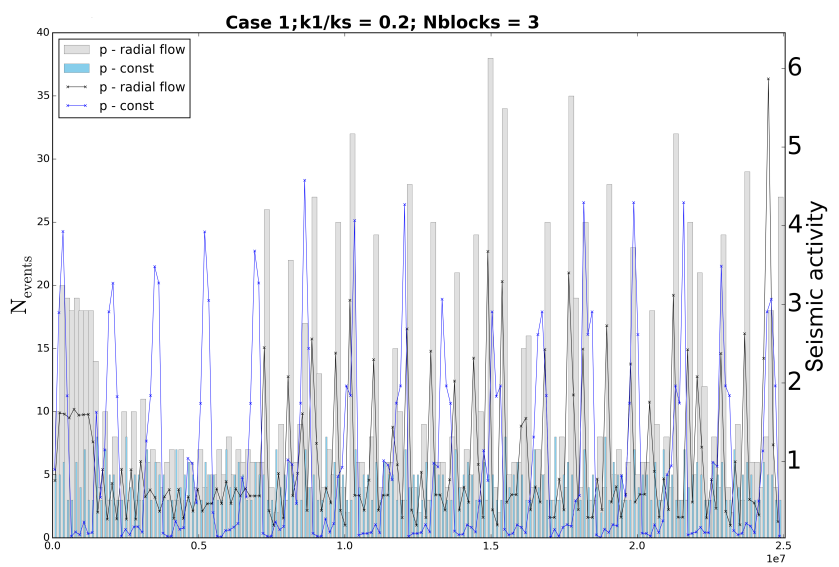

Figure 29. Time variation of seismic activity.

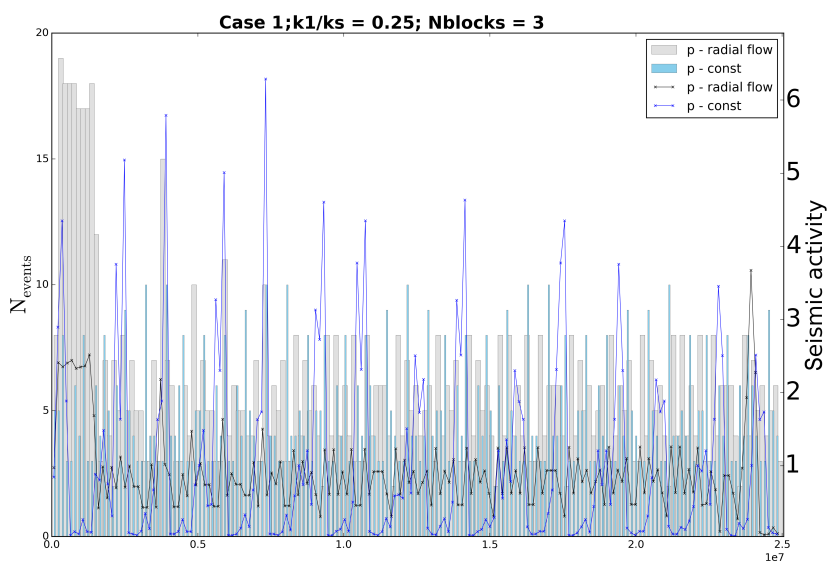

Figure 30. Time variation of seismic activity.

Case 2 is characterized by slower changes in the velocity with time than Case 1 (Fig. 26). That is why there is no clear dependence of the number of events and the block maximum velocity on the interblock link stiffness. Such behaviour becomes more evident with a decrease in parameter $A$.

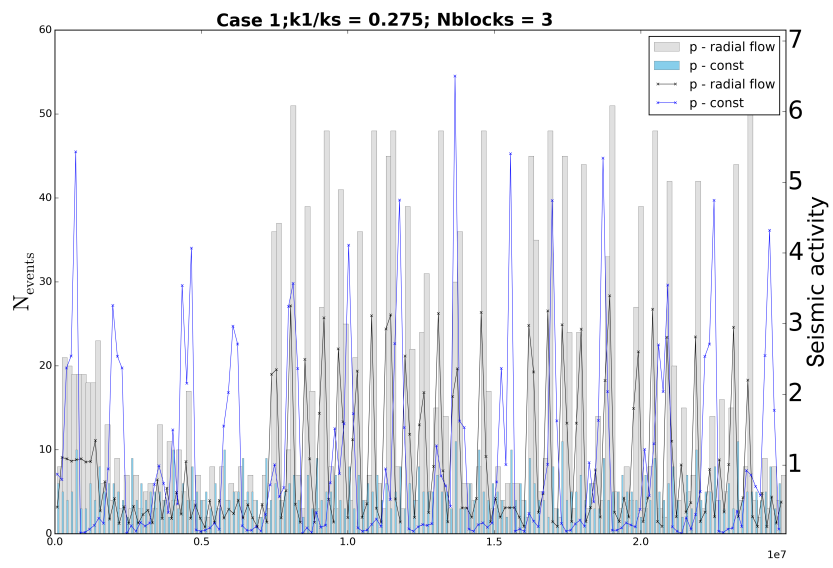

Figure 31. Time variation of seismic activity.

Our model demonstrates that the influence of the interblock link stiffness on the behaviour of the studied systems is very strong. By changing the stiffness, we may get periodic or chaotic motion of the system and occurrence of the first strong seismic event almost immediately after the injection start or after a relatively long time (compare Figs. 27 and 28); furthermore, the main seismic activity may occur at the moment of the injection start, when the pressure gradient is the highest, or in the post-injection phase. In Figs. 2730 the seismic activity variations in the form of the number of events per 10 days (left vertical axis) and the ratio of the cumulative seismic moment of events to the average cumulative seismic moment per 10 days (right vertical axis) are shown for both "natural" (Set 1) and "induced" (Set 2) seismicity. The "natural" seismic activity variations have almost the same amplitudes during all considered time intervals, while the "induced" seismic activity variations depend on interblock link stiffness: in the case of small stiffness the amplitude of the seismic activity during injection is almost the same as in the post-injection period (Fig. 29). When the stiffness becomes higher, the seismicity during injection becomes twice greater than the post-injection activity (Fig. 30); 


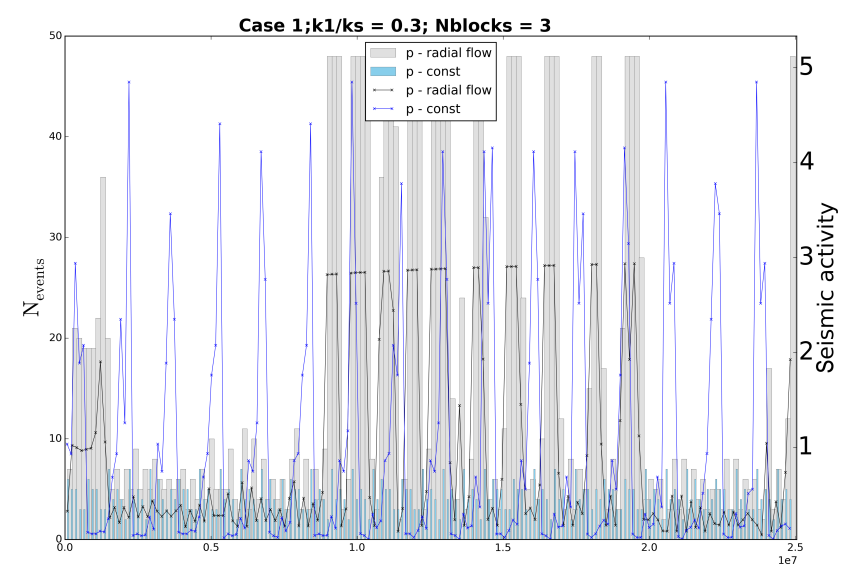

Figure 32. Time variation of seismic activity.

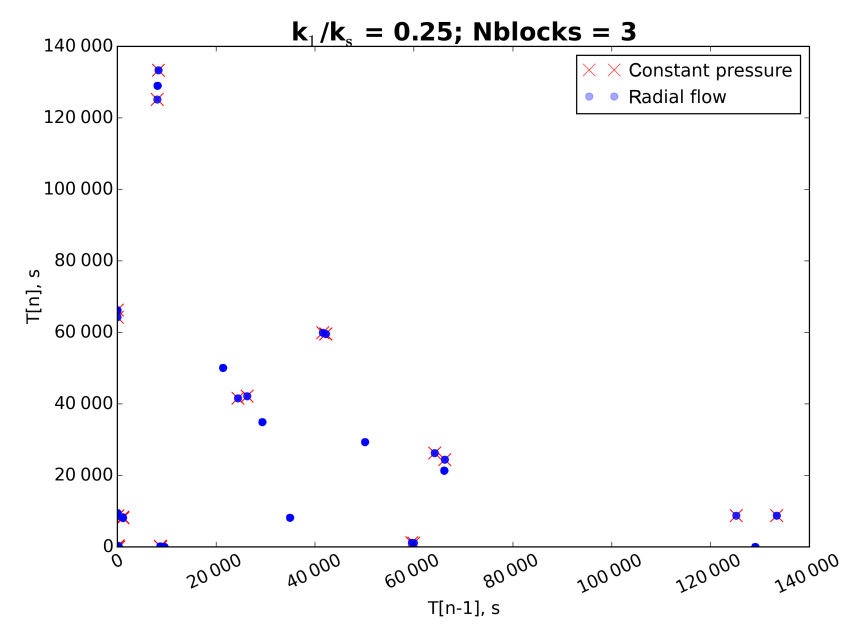

Figure 33. Iteration map of recurrence intervals of seismic events, $T_{n}$ denotes the time interval between the $n$th and $(n+1)$ th events. The map includes events that occurred at time $t \geq 8 \times 10^{6} \mathrm{~s}$.

the further increase in the interblock link stiffness leads to a significant increase in the post-injection activity (Figs. 31 and 32).

The recurrence maps of the seismic event sequences are shown in Figs. 33 and 34. It could be seen that for $k_{1} / k_{s}=0.25$ the time intervals between two events converge to several points for both "induced" and "natural" seismicity (only post-injection seismic activity is considered). For $k_{1} / k_{s}=0.3$, the "natural" seismicity shows periodic variations, while the "induced" seismicity has more complicated chaotic behaviour. For other values of $k_{1}$ both "induced" and "natural" seismicity shows the chaotic variations.

\section{Discussion and conclusions}

The problem of the influence of the fluid injection on the tectonic fault sliding and generation of the seismic events was studied by numerical calculations of the peculiarities of mo-

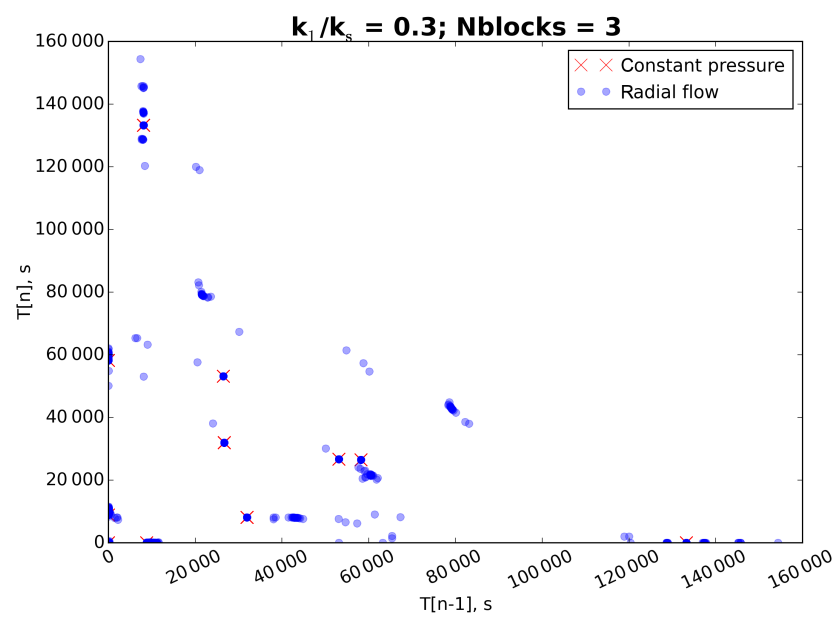

Figure 34. Iteration map of recurrence intervals of seismic events; $T_{n}$ denotes the time interval between the $n$th and $(n+1)$ th events. The map includes events that occurred at time $t \geq 8 \times 10^{6} \mathrm{~s}$.

tions of a system of blocks (consisting of up to 25 blocks) elastically connected to each other and connected by elastic springs to a constant-velocity moving driver (the multidegree-of-freedom spring-block model). The rate-and-state friction model with the two-parametric friction law was adopted for description of the friction between the blocks and the substrate. Initially the block system was in steadysliding state; then, its state was disturbed by the pore pressure increase. The influences of the model parameters (the number of the blocks, the spring stiffness, the velocity weakening parameter) on the process of the model seismicity variations were considered.

It was shown that the considered spring-block system could exhibit different types of motion with different patterns. The motion could be periodic or chaotic; the magnitude of the seismic events depends on fragmentation of the fault system (the number of blocks in the considered model) and may have different values. The analysis shows that the stiffness of the link between the blocks affects significantly the behaviour of the model and the resulting seismicity, so the main seismic activity could appear directly after the start of the fluid injection or in the post-injection phase. Such influence of the injection on seismicity could be observed in the real cases. However, the parameters in the rate-and-state model are known only from laboratory experiments, and it is hard to believe that one should use the same values to describe the real-scale phenomena. Yet our study showed that it is possible to select more suitable parameters that will allow one to match results of calculations and data of real observations. It can be concluded that the considered model has the potential to be used for the estimations of the possible fluid-induced seismicity activity variations. 
Data availability. Results of calculations are available by e-mail request.

Competing interests. The authors declare that they have no conflict of interest.

Acknowledgements. The financial support of the Russian Foundation for Basic Research (project no. 16-05-00869) is acknowledged.

Edited by: A. Dyskin

Reviewed by: two anonymous referees

\section{References}

Abe, Y. and Kato, N.: Complex Earthquake Cycle Simulations Using a Two-Degree-of-Freedom Spring-Block Model with a Rateand State-Friction Law, Pure Appl. Geophys. 170, 745-765, doi:10.1007/s00024-011-0450-8, 2013.

Abe, Y. and Kato, N.: Intermittency of earthquake cycles in a model of a three-degree-of-freedom spring-block system, Nonlin. Processes Geophys., 21, 841-853, doi:10.5194/npg-21-841-2014, 2014.

Abramovitz, M. and Stigan, I.: Handbook of special functions, M., Nauka, 56-59, 1979 (in Russian).

Brace, W. F. and Byerlee, J. D.: Stick-slip as mechanism for earthquakes, Science, 153, 990-992, doi:10.1126/science.153.3739.990, 1966.
Burridge, R. and Knopov, L.: Model and theoretical seismicity, B. Seismol. Soc. Am., 67, 341-371, 1967.

Dieterich, J. H.: Earthquake nucleation on faults with rate and state-dependent friction, Tectonophysics, 211, 115-134, doi:10.1029/96JB00529, 1992.

Dinske, C.: Interpretation of fluid-induced seismicity at geothermal and Hydrocarbon Reservoir of Basel and Cotton Valley, $\mathrm{PhD}$ Thesis, 151 pp., 2010.

Erickson, B., Birnir, B., and Lavallée, D.: A model for aperiodicity in earthquakes, Nonlin. Processes Geophys., 15, 1-12, doi:10.5194/npg-15-1-2008, 2008.

Grassberger, P. and Procaccia, I.: Measuring the strangeness of strange attractors, Physica D: Nonlinear Phenomena, 9, 189-208, doi:10.1016/0167-2789(83)90298-1, 1983.

Gu, J. C., Rice, J. R., Ruina, A. L., and Tse, S. T.: Slip motion and stability of a single degree of freedom elastic system with rateand-state dependent friction, J. Mech. Phys. Solids, 32, 167-196, doi:10.1016/0022-5096(84)90007-3, 1984.

Häring, M. O., Schanz, U., Ladner, F., and Dyer, B. C.: Characterisation of the Basel 1 enhanced geothermal system, Geothermics, 37, 469-495, doi:10.1016/j.geothermics.2008.06.002, 2008.

Hobbs, B. E.: Chaotic behaviour of frictional shear instabilities, Rockbursts and Seismicity in Mine, Mineapolis, 87-91, 1990.

Matthews, C. S. and Russel, D. G.: Pressure buildup and flow tests in wells, Monograph, Society of Petroleum Engineers of AIME, Dallas, TX, 167, 1967.

Turuntaev, S. B., Vorohobina, S. V., and Melchaeva, O. Y.: Identification of anthropogenic changes of seismic regime using methods of nonlinear dynamics, Phys. Earth, 3, 52-65, 2012. 